\title{
L5/S1 disc herniation and S1 pedicle aplasia: case report and review of the literature
}

\author{
Michael Payer • Enrico Tessitore
}

Received: 3 October 2013 / Accepted: 18 January 2014 / Published online: 8 February 2014

(C) Springer-Verlag Wien 2014

Dear editor,

Pedicle aplasia is very rare in all segments of the human spine [1-5]. Most cases of absent pedicles are asymptomatic and found incidentally on diagnostic imaging $[2,4]$. Absence of a spinal pedicle is thought to result from a large embriological retrosomatic cleft [6], occurring between the third and 20 th weeks of gestation as do most vertebro-medullary malformations [1]. Coexistence of other vertebral anomalies equally favors a congenital cause [4]: abnormality of the corresponding facets and transverse process hypoplasia may accompany larger pedicular defects [6]. Congenital pedicle absence seems to be more frequent in the cervical and thoracic than in the lumbo-sacral spine $[3,7,8]$. Pedicle aplasia has specific radiographic features: a false appearance of an enlarged neural foramen; a dysplastic, dorsally displaced ipsilateral articular pillar and lamina; and a dysplastic ipsilateral transverse process [8]. Biomechanical weakening of the affected segment has been the major concern of pedicle aplasia $[2,4]$.

We present a generally healthy 52-year-old male patient with left-sided acute $\mathrm{S} 1$ radiculopathy with sensory and motor (M3) deficit. MRI revealed a left-sided contained L5/S1 disc herniation and bilateral facet joint hypertrophy, compressing the left S1 nerve root (Fig. 1). The left neuroforamen at L5/S1 appeared very large on the preoperative MRI, and the leftsided S1 pedicle could not be identified (Fig. 1). Because of intractable radicular pain and motor weakness, microsurgical interlaminar decompression and microsurgical disc herniation

M. Payer $(\bowtie)$

Neurosurgical Department, Hirslanden Klinik, Witellikerstrasse 40,

8032 Zürich, Switzerland

e-mail: mpayer@hotmail.com

M. Payer $\cdot$ E. Tessitore

Neurosurgical Department, University of Geneva, Geneva, Switzerland removal was performed. The left S1 pedicle was not found when checking for disc fragments caudally along the S1 nerve root with a blunt nerve hook, as expected from the presurgical MRI assessment. No supplementary surgical steps were taken. Postoperatively, the patient recovered S1 muscle strength to M4-5 and was without radicular and lumbo-sacral pain at the 6- and 12-month follow-ups.

We could only identify three reports on lumbo-sacral pedicle aplasia $[2,4,6]$. Kaito et al report a congenital absence of the right L5 pedicle and contralateral L5 radicular pain from an overloaded hypertrophic facet joint in a 54-year-old man. After unsuccessful conservative treatment, the patient underwent posterior lumbar interbody fusion (PLIF) [2].

Patel et al describe a 13-year-old girl with congenital hypoplasia of the L5 pedicles and absence of the superior and inferior articulating processes, creating an abnormal facet joint between the L4 inferior articulating facet and the S1 superior articulating facet. Because of instability, posterior L4-S1 fusion was performed. They also report on a 10-yearold girl with absent left S1 pedicle and a severely degenerated hyperplastic contralateral L5-S1 facet joint with a fractured superior articulating process. Because of uncontrollable low back pain, they performed a posterior instrumented fusion with bilateral L5 pedicle screws, a right S1 pedicle screw, and a left S2 alar screw. Both patients did well at 18 months and 5 months, respectively [4].

Sener describes a 34-year-old woman with agenesis of the right $\mathrm{S} 1$ pedicle in conjunction with an anomalous L5/S1 facet and conjoined L5 and S1 nerve roots as well as developmental lumbar stenosis. The patient experienced only moderate back pain and did not need surgical intervention [6].

To our knowledge, we report the first case of sacral pedicle aplasia in association with lumbo-sacral disc herniation. In the absence of the S1 pedicle, the superior articular process of S1 is only attached to the posterior arch of S1, which in contrast to the lumbar, thoracic or cervical segments is in continuity 

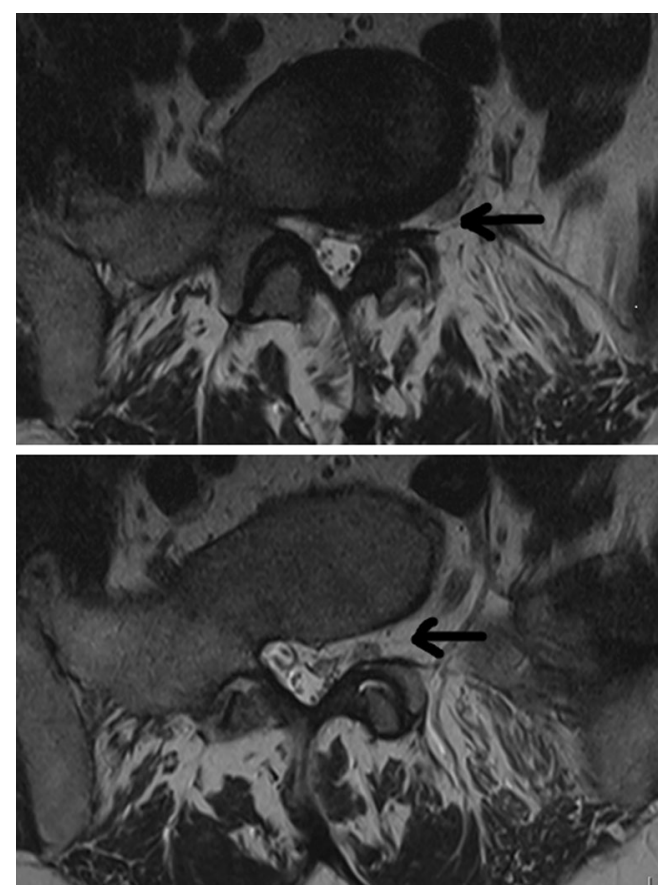

Fig. 1 Axial T2 weighted MRI-at the L5/S1 disc level (upper image) showing left-sided S1 pedicle aplasia (arrow) and S1 nerve root compression between hypertrophic facet joint and disc herniation-below the L5/ S1 disc level (lower image) showing left-sided S1 pedicle aplasia (arrow)

with all posterior elements of the sacrum and thus provides a more solid anchorage and maintains segmental stability at least partially. This may explain that our patient had a favorable outcome over the observation period of one year despite the pedicle aplasia and disc herniation removal at the same level.

Conflicts of Interest None

\section{References}

1. Geoffray A, Clément JL, Leloutre B, Albertario M, Béchard-Sevette N (2008) A case of severe congenital kyphoscoliosis secondary to multiple bilateral thoracic pedicle aplasia. Pediatr Radiol 38(7):780-2

2. Kaito T, Kato Y, Sakaura H, Yamamoto K, Hosono N (2005) Congenital absence of a lumbar pedicle presenting with contralateral lumbar radiculopathy. J Spinal Disord Tech 18(2):203-5

3. Moon SJ, Lee JK, Seo BR, Kim SH (2008) Traumatic subluxation associated with absent cervical pedicle: case report and review of the literature. Spine 33(18):E663-6

4. Patel AJ, Vadivelu S, Desai SK, Jea A (2013) Congenital hypoplasia or aplasia of the lumbosacral pedicle as an unusual cause of spondylolisthesis in the pediatric age group. J Neurosurg Pediatr 11: 717-721

5. Rauzzino MJ, Shaffrey CI, Bartkowski H, Nockels R, Wiggins G (2000) Multilevel thoracic pedicle aplasia causing congenital thoracic kyphosis: case report. Neurosurgery 46(4):988-90, discussion 990-1

6. Sener RN (1997) Sacral pedicle agenesis. Comput Med Imaging Graph 21(6):361-3

7. Sheehan J, Kaptain G, Sheehan J, Jane J Sr (2000) Congenital absence of a cervical pedicle: report of two cases and review of the literature. Neurosurgery 47(6): 1439-42

8. Wiener MD, Martinez S, Forsberg DA (1990) Congenital absence of a cervical spine pedicle: clinical and radiologic findings. AJR 155: $1037-1041$ 